\title{
Molecular Dynamics Simulation of Phase Behavior in a Bolaamphiphilic Solution*)
}

\author{
Susumu FUJIWARA, Takumi MIYATA, Masato HASHIMOTO, Yuichi TAMURA ${ }^{1)}$, \\ Hiroaki NAKAMURA $^{2)}$ and Ritoku HORIUCHI ${ }^{2)}$ \\ Kyoto Institute of Technology, Matsugasaki, Sakyo-ku, Kyoto 606-8585, Japan \\ ${ }^{1)}$ Konan University, 8-9-1 Okamoto, Higashinada-ku, Kobe 658-8501, Japan \\ 2) National Institute for Fusion Science, 322-6 Oroshi-cho, Toki 509-5292, Japan
}

(Received 26 November 2014 / Accepted 5 February 2015)

\begin{abstract}
The phase behavior of bolaamphiphilic solutions is studied by coarse-grained molecular dynamics simulations of semiflexible bolaamphiphilic molecules with explicit solvent molecules. Our simulations show that six kinds of self-assembled structures (spherical micelles, worm-like micelles, bicontinuous structure, hexagonal structure, plate-like micelles, and lamellar structure) are obtained. It is established that, at low concentrations, a plate-like micelle changes to worm-like micelles, and then to spherical micelles as the hydrophilic interaction increases. Conversely, at intermediate concentrations, a lamellar structure changes to a bicontinuous structure; it then changes to worm-like micelles or a hexagonal structure as the hydrophilic interaction increases. It is also observed that the global orientational order parameter for the end bonds of bolaamphiphilic molecules can be used to clearly distinguish between the randomly-oriented structures (the spherical micelles, the worm-like micelles, and the bicontinuous structure), the lamellar structure, the hexagonal structure, and the plate-like micelles.
\end{abstract}

(C) 2015 The Japan Society of Plasma Science and Nuclear Fusion Research

Keywords: molecular dynamics simulation, phase behavior, bolaamphiphilic solution, hydrophilic interaction, orientational order parameter

DOI: $10.1585 /$ prr.10.3401029

\section{Introduction}

The self-organization or spontaneous formation of structures in plasmas has been intensively studied to improve plasma confinement. The formation of ordered structures is a universal characteristic of nonequilibrium and nonlinear systems. We investigate self-organization in soft matter systems such as self-assembly in amphiphilic systems [1-8] and structure formation in polymeric systems [9-14] in order to explore the universal selforganizing properties of nature. Amphiphilic molecules such as lipids and surfactants are composed of both hydrophilic and hydrophobic parts. In aqueous solvents, these molecules often spontaneously self-assemble into various structures such as micelles, lamellar structures and bicontinuous structures [15-17]. Although numerous computer simulation studies have been conducted on structure formation of amphiphilic molecules, each of which consists of a hydrophilic head group and a hydrophobic tail group, there have been few theoretical and simulation studies on the structure formation of bolaamphiphilic molecules, each of which contains a hydrophobic stalk and two hydrophilic ends. As far as we know, a few dissipative particle dynamics simulations have been performed to investigate the phase behavior of a bolaamphiphilic solu-

author's e-mail: fujiwara@ kit.ac.jp

*) This article is based on the presentation at the 24th International Toki Conference (ITC24). tion [18] and the shape transformations of vesicles formed by amphiphilic triblock copolymers [19].

The purpose of this study is to clarify the effect of hydrophilicity on phase behavior in bolaamphiphilic solutions. With a view to investigating the phase behavior in bolaamphiphilic solutions at the molecular level, we perform molecular dynamics (MD) simulations of coarsegrained bolaamphiphilic molecules with explicit solvent molecules and analyze the formation processes of micelles and mesophases.

\section{Simulation Model and Method}

The computational model is similar to the one used in our previous works $[4,5]$. A bolaamphiphilic molecule is modeled as a semiflexible chain, $\mathrm{AB}_{3} \mathrm{C}$, that is composed of a hydrophobic stalk with three particles (denoted by B) and two hydrophilic ends (denoted by A and C), each of which consists of one particle. A solvent molecule is modeled as a hydrophilic particle (denoted by S). The mass of each particle is $m$.

As for nonbonded potentials, the interaction between a hydrophilic particle and a hydrophobic particle is modeled by a repulsive soft core potential

$$
U_{\mathrm{SC}}(r)=4 \varepsilon\left(\frac{\sigma_{\mathrm{SC}}}{r}\right)^{9} .
$$


The interaction between a hydrophilic end particle $\mathrm{C}$ and a solvent particle $\mathrm{S}$ is modeled by the Lennard-Jones (LJ) potential

$$
U_{\mathrm{LJ}-\mathrm{CS}}(r)=4 \varepsilon_{\mathrm{CS}}\left[\left(\frac{\sigma}{r}\right)^{12}-\left(\frac{\sigma}{r}\right)^{6}\right],
$$

and all other interactions are modeled by the $\mathrm{LJ}$ potential

$$
U_{\mathrm{LJ}}(r)=4 \varepsilon\left[\left(\frac{\sigma}{r}\right)^{12}-\left(\frac{\sigma}{r}\right)^{6}\right] .
$$

Here, $r$ is the interparticle distance and $\varepsilon_{\mathrm{CS}}$ represents the intensity of the hydrophilic interaction between a hydrophilic end particle $\mathrm{C}$ and a solvent particle $\mathrm{S}$. The parameter $\sigma_{\mathrm{SC}}$ is set to $\sigma_{\mathrm{SC}}=1.05 \sigma$, as in Ref. [20]. To avoid discontinuities in the potential energy and the force due to the potential energy cutoff, we use the shifted force variant of these nonbonded potentials

$$
V_{X}(r)=U_{X}(r)-U_{X}\left(r_{\mathrm{c}}\right)-\left.\frac{\partial U_{X}}{\partial r}\right|_{r=r_{\mathrm{c}}}\left(r-r_{\mathrm{c}}\right),
$$

where $X=\mathrm{SC}$, LJ-CS or LJ, and $r_{\mathrm{c}}$ is the cutoff distance, which is set to $r_{\mathrm{c}}=3.0 \sigma$.

As bonded potentials, we consider the bond-stretching potential

$$
U_{2}\left(d_{i}\right)=k_{2}\left(d_{i}-\sigma\right)^{2}
$$

where $k_{2}$ is the bond-stretching modulus and $d_{i}$ is the bond length between two neighboring particles along the bolaamphiphilic molecule and the bond-bending potential

$$
U_{3}\left(\theta_{i}\right)=k_{3}\left(1-\cos \theta_{i}\right)
$$

where $k_{3}$ is the bending modulus of the bolaamphiphilic molecules and $\theta_{i}$ is the tilt angle between two neighboring bonds. The parameters $k_{2}$ and $k_{3}$ are set to $k_{2}=5000 \varepsilon \sigma^{-2}$, as in Ref. [20], and $k_{3}=1.0 \varepsilon$, respectively. In the following sections, we represent dimensionless quantities by an asterisk. For example, the dimensionless quantities of the number density $\rho$, the time $t$, and the temperature $T$ are $\rho^{*}=\rho \sigma^{3}, t^{*}=t \sqrt{\varepsilon / m \sigma^{2}}$, and $T^{*}=k_{\mathrm{B}} T / \varepsilon$, respectively, where $k_{\mathrm{B}}$ is the Boltzmann constant.

Numerical integrations of the equations of motion for all particles are carried out using the velocity Verlet algorithm at constant temperature with a time step $\Delta t^{*}=$ 0.0005 , and the temperature is controlled at every 10 time steps by ad hoc velocity scaling [21]. We apply periodic boundary conditions and the number density is set to $\rho^{*}=0.75$. The total number of particles is 5832 .

Initially, we prepare homogeneous bolaamphiphilic solutions with various bolaamphiphilic concentrations $\left(c_{\mathrm{S}}=0.1,0.2, \ldots, 0.9\right)$ at high temperature $\left(T^{*}=10\right)$ for various values of the interaction parameter $\varepsilon_{\mathrm{CS}}^{*}(0.5 \leq$ $\left.\varepsilon_{\mathrm{CS}}^{*} \leq 5.0\right)$. The system is then quenched at $T^{*}=1.3$ and MD simulations of $t^{*}=2.5 \times 10^{4}\left(5.0 \times 10^{7}\right.$ time steps $)$ are performed for each simulation run.

\section{Simulation Results and Discussion 3.1 Phase behavior}

In Fig. 1, we show our simulated phase diagram pictorially. The ordinate represents the intensity of the hydrophilic interaction $\varepsilon_{\mathrm{CS}}^{*}$. The abscissa denotes the bolaamphiphilic concentration $c_{\mathrm{S}}$. Based on the direct visualizations of the obtained molecular configurations, the self-assembled structures are classified into six structures: spherical micelles, worm-like micelles, bicontinuous structure, hexagonal structure, plate-like micelles and lamellar structure. This figure shows us that, at low concentrations, a plate-like micelle changes to worm-like micelles and then to spherical micelles as the hydrophilic interaction increases. Conversely, at intermediate concentrations, it is found that the lamellar structure changes to a bicontinuous structure; it then changes to worm-like micelles or a hexagonal structure as the hydrophilic interaction increases.

\subsection{Self-assembled structure}

Snapshots of the above-mentioned six self-assembled structures formed by bolaamphiphilic molecules are shown in Fig. 2. Isosurfaces of the hydrophobic particles B, which are calculated by Gaussian splatting techniques, are depicted to show the structures clearly.

In order to distinguish the six kinds of structures, we examine the global orientational order for end bonds of the bolaamphiphilic molecules. The global orientational order parameter for end bonds $p_{2}$ is defined by

$$
p_{2}=\left\langle\frac{3 \cos ^{2} \psi-1}{2}\right\rangle_{\text {end }},
$$

where $\psi$ is the angle between two end bond vectors and $\langle\cdots\rangle_{\text {end }}$ denotes the average over all pairs of end bonds. The parameter $p_{2}$ takes a value of 1.0 when all end bonds are parallel, and it takes a value of 0.0 when end bonds are randomly oriented. In Fig. 3, we show the distribution functions of the global orientational order parameter for the end bonds of bolaamphiphilic molecules $P\left(p_{2}\right)$ for six kinds of self-assembled structures. The following char-

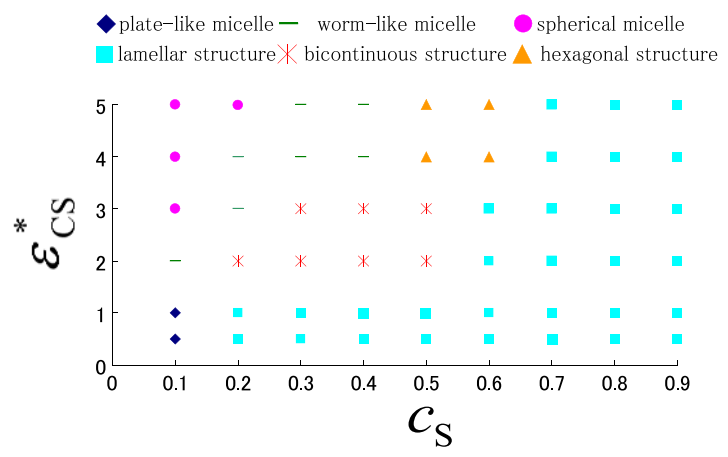

Fig. 1 Hydrophilic interaction parameter vs. bolaamphiphilic concentration phase diagram of bolaamphiphilic molecules. 
(a)

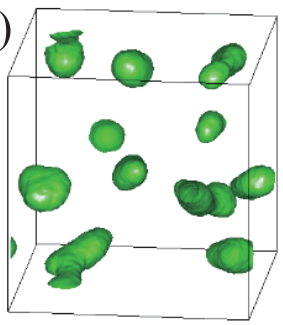

(c)



(e)

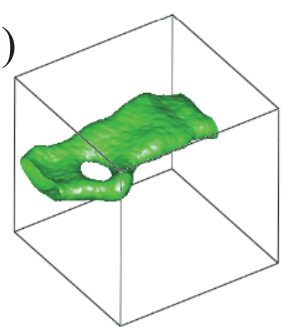

(b)

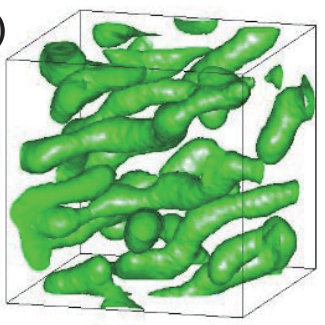

(d)

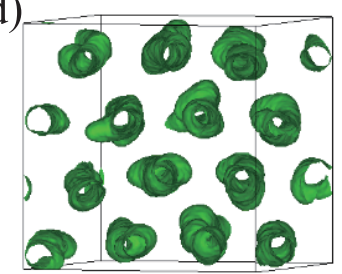

(f)

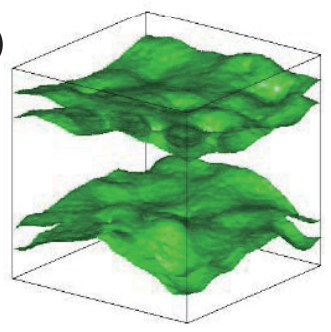

Fig. 2 Snapshots of various self-assembled structures formed by bolaamphiphilic molecules. (a) Spherical micelles, (b) worm-like micelles, (c) bicontinuous structure, (d) hexagonal structure, (e) plate-like micelles, and (f) lamellar structure.

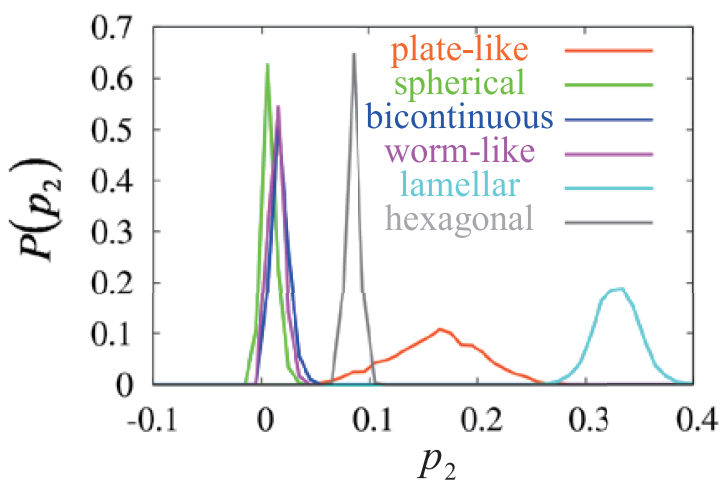

Fig. 3 Distribution function of the global orientational order parameter for the end bonds of the bolaamphiphilic molecules $P\left(p_{2}\right)$ for six kinds of self-assembled structures.

acteristic features are found from this figure. (i) The end bonds of the bolaamphiphilic molecules are randomly oriented in the case of the spherical micelles, the worm-like micelles, and the bicontinuous structure. (ii) In the case of the lamellar structure, the peak position is larger than 0.3 . (iii) In the case of the hexagonal structure, the peak position is located around 0.1. (iv) In the case of the platelike micelles, the distribution $P\left(p_{2}\right)$ becomes broad and the peak position is located around 0.15 . These results indicate
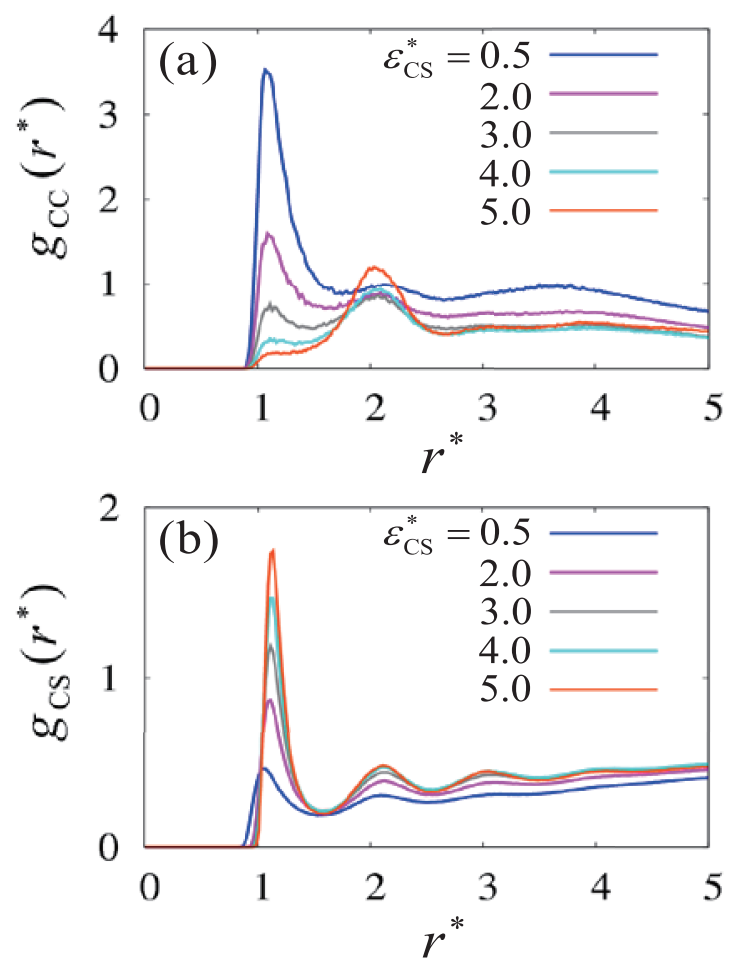

Fig. 4 The radial distribution function (a) between hydrophilic particles $\mathrm{C}, g_{\mathrm{CC}}\left(r^{*}\right)$, and (b) between hydrophilic particles $\mathrm{C}$ and solvent particles $\mathrm{S}, g_{\mathrm{CS}}\left(r^{*}\right)$, in the case of $c_{\mathrm{S}}=0.1$.

that the global orientational order parameter for end bonds of the bolaamphiphilic molecules can be used to distinguish between the randomly-oriented structures (the spherical micelles, the worm-like micelles, and the bicontinuous structure), the lamellar structure, the hexagonal structure, and the plate-like micelles.

\subsection{Radial distribution function}

Here, we investigate the radial distribution function with respect to the hydrophilic end particle C. In Fig. 4, we show the radial distribution function between hydrophilic particles $\mathrm{C}, g_{\mathrm{CC}}\left(r^{*}\right)$, and between hydrophilic particles $\mathrm{C}$ and solvent particles $\mathrm{S}, g_{\mathrm{CS}}\left(r^{*}\right)$, in the case of $c_{\mathrm{S}}=0.1$. We realize from Fig. 4 that, as $\varepsilon_{\mathrm{CS}}^{*}$ increases, the height of the first peak of $g_{\mathrm{CC}}\left(r^{*}\right)$ decreases (Fig. 4 (a)) whereas that of $g_{\mathrm{CS}}\left(r^{*}\right)$ increases (Fig. $4(\mathrm{~b})$ ). This fact indicates that, as $\varepsilon_{\mathrm{CS}}^{*}$ increases, the solvent particles $\mathrm{S}$ tend to be incorporated in between the hydrophilic end particles $\mathrm{C}$, which suggests that the local curvature of the hydrophobic stalksolvent interface tends to become larger as $\varepsilon_{\mathrm{CS}}^{*}$ increases.

\section{Conclusions}

In conclusion, we have obtained the following new results by performing MD simulations of semiflexible bolaamphiphilic molecules with explicit solvent molecules and analyzing the formation processes of micelles and mesophases. (1) Six kinds of self-assembled structures (spherical micelles, worm-like micelles, bicontin- 
uous structure, hexagonal structure, plate-like micelles and lamellar structure) are formed at a low temperature by quenching from a random configuration of bolaamphiphilic molecules in a solution at a high temperature. (2) At low concentrations, a plate-like micelle changes to worm-like micelles and then to spherical micelles as the hydrophilic interaction increases. (3) Conversely, at intermediate concentrations, the lamellar structure changes to a bicontinuous structure; it then changes to worm-like micelles or a hexagonal structure as the hydrophilic interaction increases. (4) The randomly-oriented structures (the spherical micelles, the worm-like micelles, and the bicontinuous structure), the lamellar structure, the hexagonal structure, and the plate-like micelles are clearly distinguishable by the global orientational order parameter for end bonds of bolaamphiphilic molecules. (5) The solvent particles tend to be incorporated in between the hydrophilic end particles as the hydrophilic interaction increases.

In our future work, we will study the formation of vesicles and nanotubes in a bolaamphiphilic solution by coarse-grained MD simulations.

\section{Acknowledgment}

This study was partially supported by the NIFS Collaborative Research Program (NIFS14KNTS035). The authors thank the Supercomputer Center, Institute for Solid State Physics, University of Tokyo for the facilities provided.

[1] S. Fujiwara, M. Hashimoto and T. Itoh, J. Plasma Phys. 72, 1011 (2006).
[2] S. Fujiwara, T. Itoh, M. Hashimoto and Y. Tamura, Mol. Simul. 33, 115 (2007).

[3] S. Fujiwara, T. Itoh, M. Hashimoto and R. Horiuchi, J. Chem. Phys. 130, 144901 (2009).

[4] S. Fujiwara, T. Itoh, M. Hashimoto, H. Nakamura and Y. Tamura, Plasma Fusion Res. 5, S2114 (2010).

[5] S. Fujiwara, T. Itoh, M. Hashimoto, Y. Tamura, H. Nakamura and R. Horiuchi, Plasma Fusion Res. 6, 2401040 (2011).

[6] S. Fujiwara, D. Funaoka, T. Itoh and M. Hashimoto, Comput. Phys. Commun. 182, 192 (2011).

[7] S. Fujiwara, M. Hashimoto, T. Itoh and R. Horiuchi, Chem. Lett. 41, 1038 (2012).

[8] S. Fujiwara, M. Hashimoto, Y. Tamura, H. Nakamura and R. Horiuchi, Plasma Fusion Res. 9, 3401067 (2014).

[9] S. Fujiwara and T. Sato, J. Chem. Phys. 107, 613 (1997).

[10] S. Fujiwara and T. Sato, J. Chem. Phys. 114, 6455 (2001).

[11] S. Fujiwara and T. Sato, Phys. Rev. Lett. 80, 991 (1998).

[12] S. Fujiwara and T. Sato, J. Chem. Phys. 110, 9757 (1999).

[13] S. Fujiwara and T. Sato, Comput. Phys. Commun. 142, 123 (2001).

[14] S. Fujiwara, M. Hashimoto, T. Itoh and H. Nakamura, J. Phys. Soc. Jpn. 75, 024605 (2006).

[15] J.N. Israelachvili, Intermolecular and Surface Forces (Academic Press, London, 1992) 2nd ed.

[16] Micelles, Membranes, Microemulsions, and Monolayers, edited by W.M. Gelbart, A. Ben-Shaul and D. Roux (Springer-Verlag, New York, 1994) pp.1-104.

[17] I.W. Hamley, Introduction to Soft Matter (J. Wiley, Chichester, 2007) Rev. ed.

[18] R. Shirasaki, Y. Yoshikai, H. Qian, S. Fujiwara, Y. Tamura and H. Nakamura, Plasma Fusion Res. 6, 2401116 (2011).

[19] X. Li, I.V. Pivkin, H. Liang and G.E. Karniadakis, Macromolecules 42, 3195 (2009).

[20] R. Goetz and R. Lipowsky, J. Chem. Phys. 108, 7397 (1998).

[21] M.P. Allen and D.J. Tildesley, Computer Simulation of Liquids (Clarendon, Oxford, 1987). 